Research paper

\title{
Supercritical flow profile improvement by means of a convex corner at a bend inlet
}

\author{
MOHAMMAD REZA JAEFARZADEH (IAHR Member), Professor, Civil Engineering Department, School of Engineering, \\ Ferdowsi University of Mashhad, Wakil Abad Blvd., Mashhad, PO Box 91775-1111, Iran. \\ Email: jafarzad@um.ac.ir (author for correspondence)
}

\author{
ALI REZA SHAMKHALCHIAN, MSc Student, Civil Engineering Department, School of Engineering, Ferdowsi University of \\ Mashhad, Wakil Abad Blvd., Mashhad, PO Box 91775-1111, Iran. \\ Email: shamkhalchian_alireza@yahoo.com
}

MAHBOOBEH JOMEHZADEH, MSc Student, Civil Engineering Department, School of Engineering, Ferdowsi University of Mashhad, Wakil Abad Blvd., Mashhad, PO Box 91775-1111, Iran.

Email: mahboobeh_jomezadeh@yahoo.com

\begin{abstract}
A novel method for the reduction of wave height along the outer wall of a bend is introduced by adding a convex corner to the inner bend wall. The negative waves emitted from the convex corner are then superimposed on the positive waves caused by the outer bend wall. The interaction of positive and negative waves transforms the original single-humped wave along the outer wall to a lower double-humped wave. The optimum expansion angle of the convex corner is a function of the offset width to the bend width, the radius of curvature to the bend width, and the approach flow Froude number. Numerical studies indicate that the convex corner may be used in a wide range of safe conditions free from cavitation damage. By using an optimized convex corner, the wave height may be reduced between 10 and $45 \%$.
\end{abstract}

Keywords: Bend; convex corner; oblique wave; Roe's method; supercritical flow; transition

\section{Introduction}

Cross-waves are generated in a supercritical flow channel due to side wall deflection or curvature. Concave corners generate positive waves or shocks, whereas convex corners emit negative waves or centred depressions. In a contraction, the shocks and depressions interact generating a complex wave pattern. However, if the transition is designed properly, the wave interaction subsides the wave progression in the downstream channel. The analytical solution for the wave development in straight transitions is provided by Henderson (1966) or Chow (1959). In the recent years, however, advanced numerical methods have been applied for studying shock waves in transitions (Causon et al. 1999, Jan et al. 2009).

Similarly, if a supercritical flow enters a curved channel, a group of oblique waves is produced. Their general configuration, position, and height were studied by von Kármán (1938), Knapp and Ippen (1938), and by Ghaeini Hessaroeyeh and Tahershamsi (2009) proposing analytical relationships for the extremum wave heights and locations. Supercritical flow in bends has also been studied experimentally (Reinauer and Hager 1997) and numerically (Valiani and Caleffi 2005).

Usually, the generation and development of supercritical waves in open channels and bends are not desirable from an engineering point of view because wave crests may become much higher than the inflow depth so that the wall height increases considerably. Moreover, these waves extend into the downstream channel and disturb the water surface. Ippen (1936) reported that a flood occurred on 1 January 1934 in a rectangular cross-section of a concrete channel bend of Verdugo Wash, a tributary of Los Angeles River in California, with a radius of $182.9 \mathrm{~m}$, a width of $1.5 \mathrm{~m}$, and a bend angle of $12^{\circ}$ that had an average inflow depth and velocity of $1.13 \mathrm{~m}$ and $11.6 \mathrm{~m} / \mathrm{s}$, respectively. The maximum depth was $1.77 \mathrm{~m}$ at the bend outlet. Knapp (1951) was the first to suggest several possible techniques for shock wave treatment in supercritical bend flows. However, Vischer and Hager (1994) identified six basic methods for shock wave reduction, namely chute banking, wave interference, chute vanes, bottom drops, cover plates, and reduction of the shock number. Wave interference was defined as creating negative waves of intensity

Revision received 5 August 2012/Open for discussion until 30 June 2013. 
equal to the positive disturbances. This method was proposed for chute contractions. Later on, Reinauer and Hager (1996) introduced a pyramid-shaped diffractor element to reduce shocks in contractions. In addition, they experimentally demonstrated that the previous analytical solutions based on the wave interference principle does not work for chute contractions mainly because a shock wave has a finite wavelength in the order of a flow depth. Moreover, a minor deviation from the design conditions of Ippen and Dawson (1951) leads to the development of shock waves implying that the design is highly inflexible and may work for only one discharge (Hager 2010).

In a laboratory study, Beltrami et al. (2007) extended the idea of superposition of shocks and depressions to curved channels by installing water flaps upstream of a channel bend. These generate counter-phase positive waves that superimpose on the bend negative waves caused by the inner wall and reduce the wave height.

Herein, the design of a small convex corner at a bend inlet is proposed to reduce negative disturbances, imposed on the positive waves generated along the outer wall. A convex corner reduces a single-humped wave along the outer wall to a doublehumped wave, thereby decreasing the wave height considerably. This method is simple, practical, and inexpensive. The approximate Riemann solver of Roe is applied to optimize the geometry. The robustness of the numerical method is confirmed by examining its performance in straight transitions by comparing it with the available analytical solutions and the experimental data.

\section{Hydraulics of supercritical bend flow}

If a supercritical flow enters a rectangular channel bend of central radius $r_{c}$, two sets of superficial oblique disturbances develop due to sidewall curvature (Fig. 1). Positive disturbances along the outer concave wall increase the flow depth and negative waves along the inner convex wall decrease it. The first wave crest (trough) is located at the angle $\theta_{\max }\left(\theta_{\min }\right)$ from the bend inlet along the outer (inner) wall. It is assumed that $\theta_{\max }=\theta_{\min }=\theta_{m}$ and the extreme wave positions result from the bend geometry and the approach flow characteristics as (Knapp 1951,

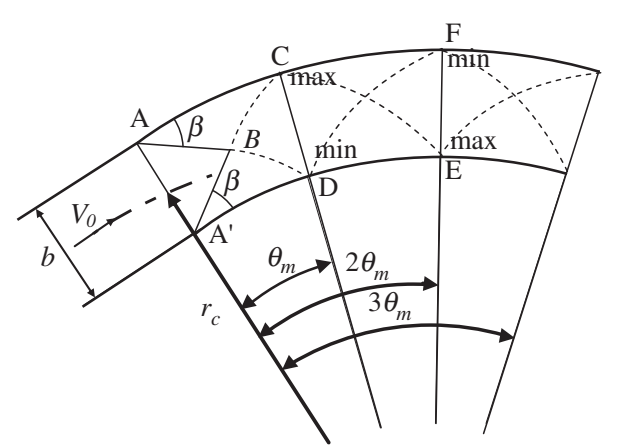

Figure 1 Development of oblique waves in a bend (Chow 1959)
Chow 1959)

$$
\theta_{m}=\tan ^{-1} \frac{2 b}{\left(2 r_{c}+b\right) \tan \beta}
$$

where $b$ is the channel width and the wave angle $\beta$ is a function of the approach flow Froude number

$$
\beta=\sin ^{-1}\left(\frac{1}{\mathrm{~F}_{\mathrm{o}}}\right), \quad \mathrm{F}_{\mathrm{o}}=\frac{V_{o}}{\sqrt{g h_{o}}}
$$

In Eq. (2), $h_{o}$ and $V_{o}$ are the depth and velocity at the bend inlet and $g$ the acceleration due to gravity. After the formation of the first crest (trough) at $\theta_{m}$, a set of consecutive wave extrema are formed along the bend walls at $2 \theta_{m}, 3 \theta_{m}$, etc.

Knapp and Ippen (1938) proposed the following relationship for the wall surface profiles:

$$
\frac{h}{h_{o}}=\mathrm{F}_{\mathrm{o}}^{2} \sin ^{2}\left(\beta \pm \frac{\theta}{2}\right) \quad 0<\theta<\theta_{m}
$$

where $h$ is the variable flow depth versus the angle $\theta$. Using Eqs. (1) - (3) the position and the crest height of the first wave at the outer wall may be determined.

\section{Performance of a convex corner at a bend inlet}

In Fig. 2, a convex corner is shown, at a bend inlet, with a deflection angle $\alpha$, an offset width $d$ and an offset length $l=d / \tan \alpha$. The corner emanates negative waves which are confined in a fan-shaped region (Fig. 2). These waves may be adjusted carefully, by changing the geometry, to interfere with the first positive wave along the outer bend wall to reduce its height. The reduction of the first peak results in the lowering of the succeeding wave heights; improving the flow conditions in the curved reach, and smoothing the wavy water surface in the downstream channel. Predicting the first crest location and water surface elevation is necessary for designing the wall height.

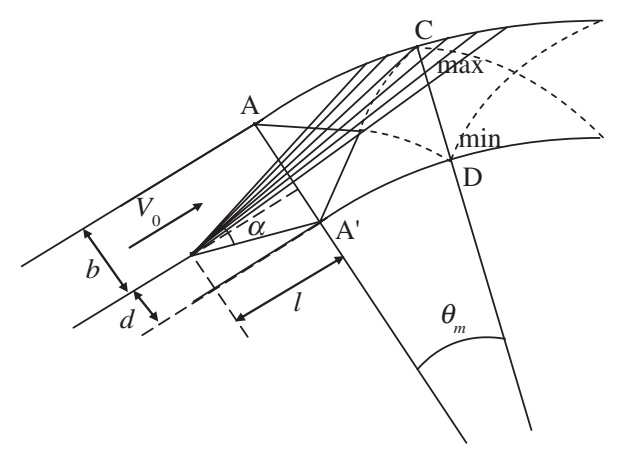

Figure 2 Interaction of centered depression and oblique shock in a bend 


\section{Numerical approach}

\subsection{Shallow water equations}

The shallow water equations (SWE) are used to analyse hypercritical flows $\left(F_{\circ}>3\right)$. Their depth-averaged differential form upon neglecting frictional and turbulence effects in a horizontal channel is (Toro 2001)

$$
\frac{\partial \boldsymbol{U}}{\partial t}+\frac{\partial \boldsymbol{F}}{\partial x}+\frac{\partial \boldsymbol{G}}{\partial y}=0
$$

where $\boldsymbol{U}$ is the vector of conservative variables and $\boldsymbol{F}(\boldsymbol{G})$ is the flux vector in the $x(y)$ coordinate system defined as

$$
\begin{aligned}
\boldsymbol{U} & =\left[\begin{array}{c}
h \\
h u \\
h v
\end{array}\right], \quad \boldsymbol{F}(\boldsymbol{U})=\left[\begin{array}{c}
h u \\
h u^{2}+\frac{1}{2} g h^{2} \\
h u v
\end{array}\right], \\
\boldsymbol{F}(\boldsymbol{U}) & =\left[\begin{array}{c}
h v \\
h u v \\
h v^{2}+\frac{1}{2} g h^{2}
\end{array}\right]
\end{aligned}
$$

where $(u, v)$ are the depth-averaged velocities in the $(x, y)$ directions.

\subsection{Two-dimensional numerical scheme of Roe}

The SWE are solved using the approximate Riemann solver of Roe over a quadrangular-structured grid. This is a Godunov-type high-resolution scheme able to simulate shock and depression waves in supercritical flows. A minmod limiter is implemented to control wiggles at the vicinity of shocks (Leveque 2002). For two-dimensional supercritical flows, three boundary conditions need to be specified at the upstream boundary but none at the downstream boundary (Chaudhry 2008). Therefore, the flow depth $h$ and the velocities $u$ and $v$ are set equal to their approach flow values at the upstream boundary, and are fixed during the computations. At the downstream end, the dependent variables are interpolated from the solution domain. The solid boundaries are simulated assuming slip boundary conditions. The steady supercritical flow with undisturbed flow depths and velocities are introduced to the model as initial conditions. The computational time step is controlled by the Courant-Friedrichs-Lewy (CFL) stability criterion. The unsteady flow equations are integrated in time to converge to a steady-state solution if the absolute sum of the depth residuals is less than, say, $10^{-7}$ (Shamkhalchian and Jaefarzadeh 2011). To verify the robustness of the numerical scheme, oblique shock waves and centred depressions produced by supercritical flow in straight transitions were modelled and compared with the analytical and numerical results of other researchers.

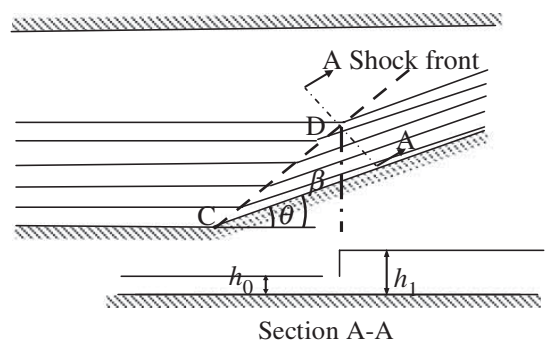

Figure 3 Oblique shockwave in a concave corner

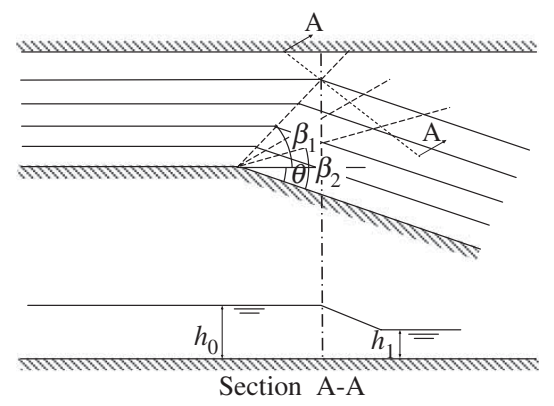

Figure 4 Centered depression in a convex corner

\subsection{Oblique shock waves}

Consider a concave corner as shown in Fig. 3. The length and width of the inlet channel are 1.4 and $0.84 \mathrm{~m}$ and the contraction angle is $15^{\circ}$. The upstream flow depth is $1 \mathrm{~m}$ and the approach flow Froude number is $F_{0}=2$. This corner was modelled by Causon et al. (1999) using the Harten, Lax, and van Leer (HLL) approximate Riemann solver over a boundary-fitted grid with $84 \times 140$ cells. The results of Roe's scheme having the same cell number and size compare excellently with the analytical solution (Chow 1959) and the numerical results of the model of Causon et al. (1999) for the downstream Froude number and depth. However, the numerical shock front angle is underestimated by $0.55^{\circ}$ as compared with the analytical solution. Note that the flow depth increases by $54 \%$ beyond the oblique shock.

\subsection{Centred depression waves}

Assume a convex corner with an expansion angle of $15^{\circ}$ (Fig. 4). The geometrical characteristics of the upstream channel and the number and size of the computational cells are identical to the previous example, except for $F_{0}=1.95$. The results of Roe's scheme in comparison with the analytical solution and the numerical model of Causon et al. (1999) for the downstream Froude number, flow depth, and depression angles $\beta_{1}$ and $\beta_{2}$, measured with reference to the approach flow and final flow directions are quite close to each other and the downstream flow depth is reduced by $51 \%$. 


\section{Numerical modelling of supercritical bend flow}

Reinauer and Hager (1997) studied experimentally supercritical flow in rectangular channel bends. The geometrical characteristics of one of their horizontal-curved channels were: bend radius $=3.607 \mathrm{~m}$, bend angle $=51^{\circ}$, and channel width $=$ $0.25 \mathrm{~m}$. Based on their observations, the following relationships for the location of the first wave crest and the wave profile along the outer wall are

$$
\begin{aligned}
\tan \theta_{\text {max }} & = \begin{cases}\mathrm{F}_{\mathrm{o}} \frac{b}{r_{c}}, & \mathrm{~F}_{\mathrm{o}} \frac{b}{r_{c}} \leq 0.35 \\
0.6 \sqrt{\mathrm{F}_{\mathrm{o}} \frac{b}{r_{c}}}, & \mathrm{~F}_{\mathrm{o}} \frac{b}{r_{c}}>0.35\end{cases} \\
\tau_{o w} & =\sin ^{1.5}\left(\frac{\pi}{2} \frac{\theta}{\theta_{\text {max }}}\right) \quad 0 \leq \frac{\theta}{\theta_{\text {max }}}<1.25
\end{aligned}
$$

wih the normalized wall profile

$$
\tau_{o w}=\frac{h-h_{o}}{h_{\max }-h_{o}}
$$

where $h_{\max }$ is the maximum flow depth. Equation (7) applies for $0.75<\theta / \theta_{\max } \leq 1.25$.

Beltrami et al. (2007) suggested an alternative surface profile along the outer wall as

$$
\tau_{o w}=\frac{1-J_{o}\left(3.8\left(\theta / \theta_{\max }\right)\right)}{1.4}
$$

where $J_{o}$ is the Bessel function of the first kind of order zero. Equation (9) is close to Eq. (7) if $0.75<\theta / \theta_{\max } \leq 1.25$ and gives appropriate results if $1.25<\theta / \theta_{\max } \leq 2$. Valiani and Caleffi (2005) modelled the channel bend of Reinauer and Hager using the HLL solver. Herein, this bend was simulated using the Roe solver with quadrangular cells. In Fig. 5, the numerical results for the normalized wall profile $\tau_{o w}\left(\theta / \theta_{\max }\right)$ are in close agreement with Eqs. (7) and (9) for different $F_{0}$. In Fig. 6, the maximum dimensionless depth $\eta=h_{\max } / h_{o}$ obtained from the

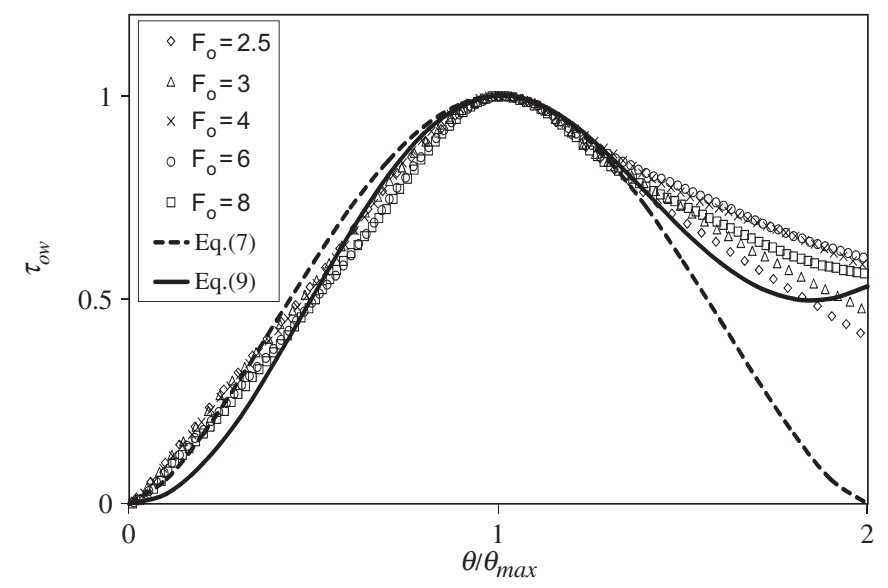

Figure 5 Normalized wall profile $\tau_{o w}\left(\theta / \theta_{\max }\right)$

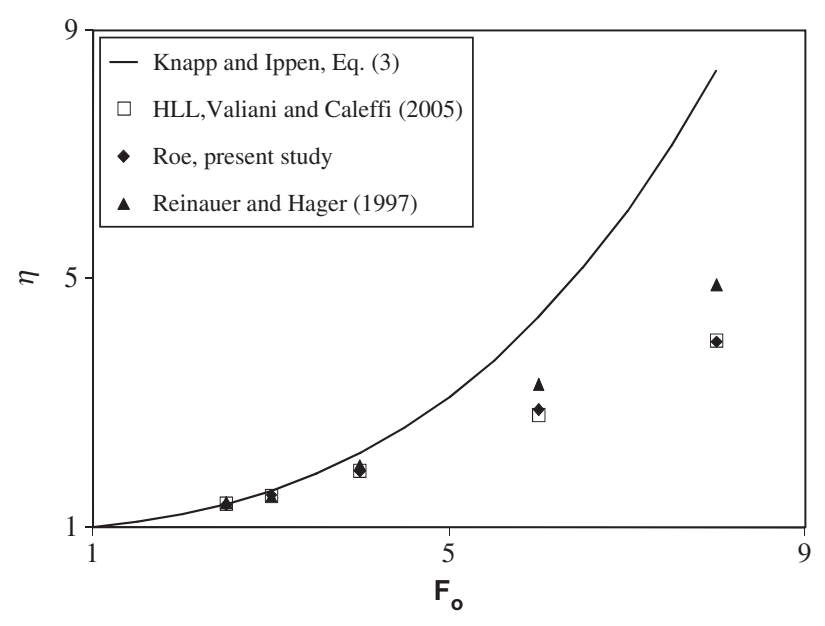

Figure 6 Maximum dimensionless depth $\eta\left(\mathrm{F}_{\mathrm{o}}\right)$

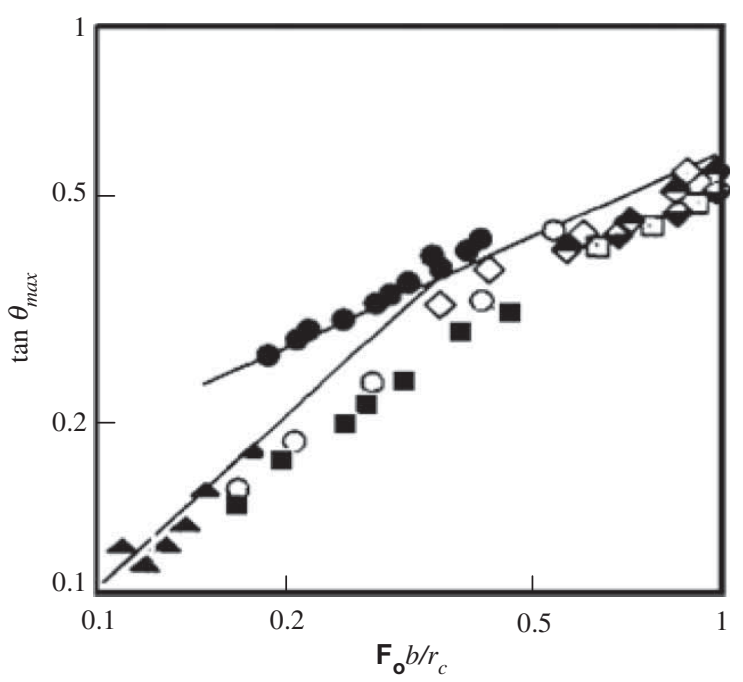

Figure 7 Crest location $\tan \theta_{\max }$ versus $\mathrm{F}_{\mathrm{o}} b / r_{c}$, (-) Eqs. (6), present numerical experiments, all other data by Reinauer and Hager (1997)

analytical equation of Knapp and Ippen (1938), the numerical schemes of Roe and HLL, and the experiments of Reinauer and Hager (1997) are plotted against $\mathrm{F}_{\mathrm{o}}$, for $h_{o}=5 \mathrm{~cm}$. The numerical results of Roe and HLL are nearly identical. For $F_{0} \leq 4.5$, the analytical and numerical results are close to the experimental data, whereas for $F_{0}>4.5$ the numerical results, particularly the analytical solutions, are far from the tests. Based on numerical experiences for $F_{0}>4.5$, a zone of separation occurs in the vicinity of the inner bend wall (Shamkhalchian and Jaefarzadeh 2011), as was also reported by Reinauer and Hager (1997). Figure 7 refers to the location of first wave crest $\tan \theta_{\max }$ against $\mathrm{F}_{\mathrm{o}} b / r_{c}$, obtained from the present numerical experiments together with Eqs. (6) and data provided by Reinauer and Hager (1997). The numerical and experimental data generally agree.

\section{Numerical studies of convex corner in bend flow}

From the analytical equations (1) - (3) and the experimental equations (6)-(9), it is deduced that the normalized wall profile in bend 


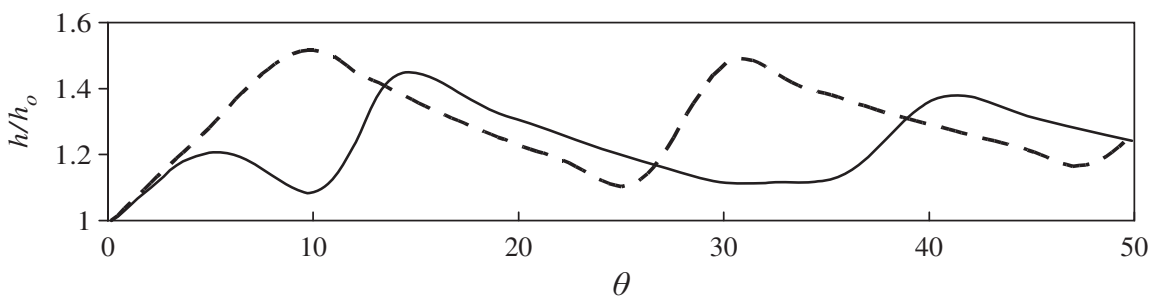

Figure 8 Dimensionless outer wall wave profile $h(\theta) / h_{o}$ for $r_{c} / b=15, \mathrm{~F}_{\mathrm{o}}=3, d / b=0.12$ and $\tan \alpha=0.1$, ( $\longrightarrow$ ) bend without convex corner, (-) bend with convex corner
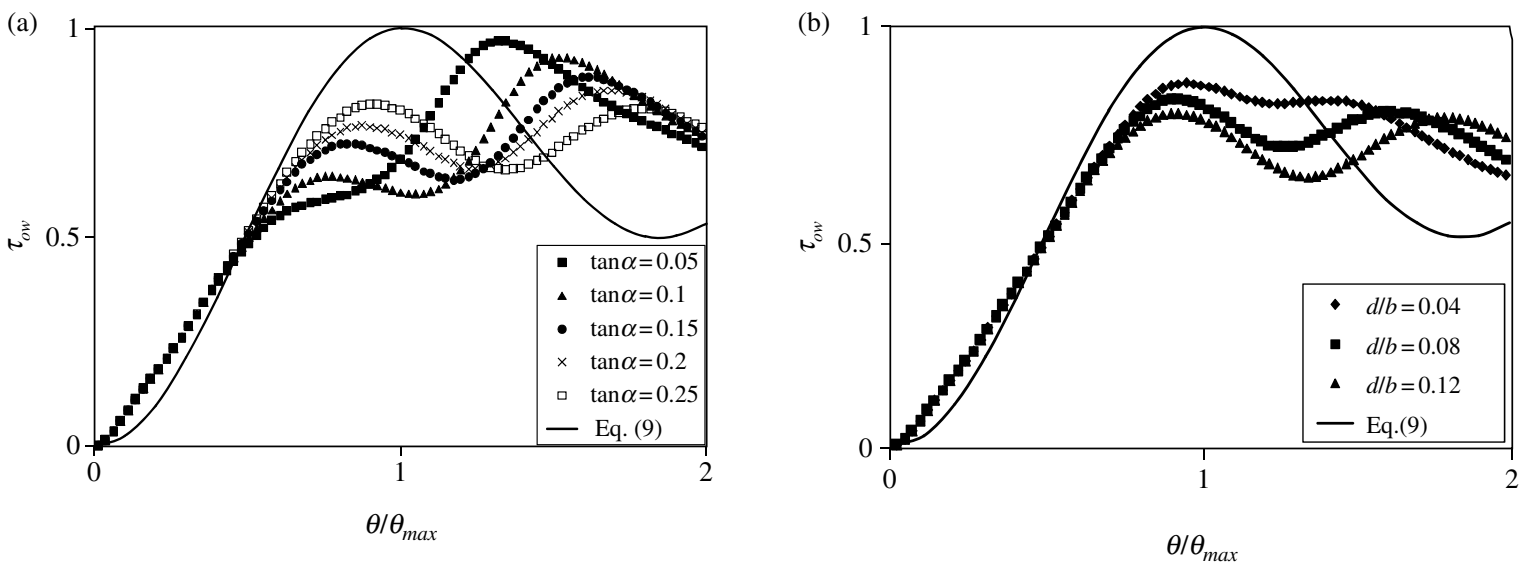

Figure 9 Normalized wall profiles $\tau_{o w}\left(\theta / \theta_{\max }\right)$ for $r_{c} / b=15$ and $\mathrm{F}_{\mathrm{o}}=4.5$. (a) $d / b=0.12$ (b) $\tan \alpha=0.25$

flow is a function of $\mathrm{F}_{\mathrm{o}}$ and $r_{c} / b$. In the presence of a convex corner, the geometrical characteristics of the corner as shown in Fig. 2 will affect the profile as well, so

$$
\tau_{o w}=f\left(\mathrm{~F}_{\mathrm{o}}, \frac{r_{c}}{b}, \alpha, \frac{d}{b}\right)
$$

The channel bend of Reinauer and Hager (1997) was considered with $r_{c} / b=15$ and two hypothetical bends with $r_{c} / b=10$ and $r_{c} / b=20$. The inlet channel width was fixed at $b=0.25 \mathrm{~m}$. Roe's model, a powerful tool for simulating shocks and depressions in transitions, was used to study the performance of the convex corner in bends. Herein, this bend was simulated with a maximum number of 3740 quadrangular cells. The grid sizes varied from $2 \mathrm{~mm} \times 5 \mathrm{~mm}$ around the convex corner tip to $20 \mathrm{~mm} \times 25 \mathrm{~mm}$ along the outer bend wall. The computational time step was of the order $0.5 \times 10^{-5}$ s to satisfy the CFL stability criterion.

In Fig. 8, the dimensionless outer wall profile $h / h_{o}$ is plotted versus $\theta$, with and without the convex corner for $r_{c} / b=15$, $\mathrm{F}_{\mathrm{o}}=3, d / b=0.12$ and $\tan \alpha=0.1$. The presence of the corner transforms the first single-humped wave into a double-humped wave, thus reducing the crest height of subsequent waves.

In Fig. 9(a), the normalized wall profile with $r_{c} / b=15$, $\mathrm{F}_{\mathrm{o}}=4.5$ and $d / b=0.12$ is shown for various $\alpha$. By increasing the expansion angle, the heights of the two humps are close to each other so that at $\tan \alpha=0.25$ the humps are nearly equal. In Fig. 9(b), the normalized wall profiles for $r_{c} / b=15, \mathrm{~F}_{\mathrm{o}}=4.5$ and $\tan \alpha=0.25$ are shown for different $d / b$ ratios.
By increasing $d / b$, the crest height of the double-humped wave decreases. Numerical experiments indicate that changing the offset width and expansion angle of the convex corner relocates the position and height of the double-humped wave. For a certain $d / b$, the optimum expansion angle $\alpha_{\text {opt }}$ results as the heights of the two humps are identical associated with the maximum reduction of the wall profile. The length of transition may be determined when the optimum angle is achieved.

The optimum expansion angle was estimated in a series of numerical studies for $d / b=0.04-0.12, \tan \alpha=0.15-0.4, \mathrm{~F}_{\mathrm{o}}=$ 2.5-4.5 and $r_{c} / b=10-20$. Figure 10 shows tan $\alpha_{\text {opt }}$ versus $\mathrm{F}_{\mathrm{o}}$ for different values of $r_{\mathrm{c}} / b$. In these graphs, solid lines are best fitted to all sets of data associated with various $d / b$. For $\mathrm{F}_{\mathrm{o}} \leq 3$, the optimum angle does not depend on the approach flow Froude number. For $F_{0}>3$, it decreases linearly and the slope of the lines decreases as $r_{\mathrm{c}} / b$ increases.

The following equations describe the solid lines

$$
\tan \alpha_{\text {opt }}=\left\{\begin{array}{l}
{[4.2784(d / b)+0.7633] \frac{1}{\left(r_{c} / b\right)}} \\
\quad+0.0479(d / b)+0.259, \quad \mathrm{~F}_{\mathrm{o}} \leq 3 \\
{\left[4.2784(d / b)+0.7633+1.7323\left(3-\mathrm{F}_{\mathrm{o}}\right)\right]} \\
\frac{1}{\left(r_{c} / b\right)} \\
-0.0451\left(3-\mathrm{F}_{\mathrm{o}}\right)+0.0479(d / b)+0.259, \mathrm{~F}_{\mathrm{o}}>3
\end{array}\right.
$$

The maximum and minimum errors of Eqs. (11) are 7.34 and $0.17 \%$, respectively. 

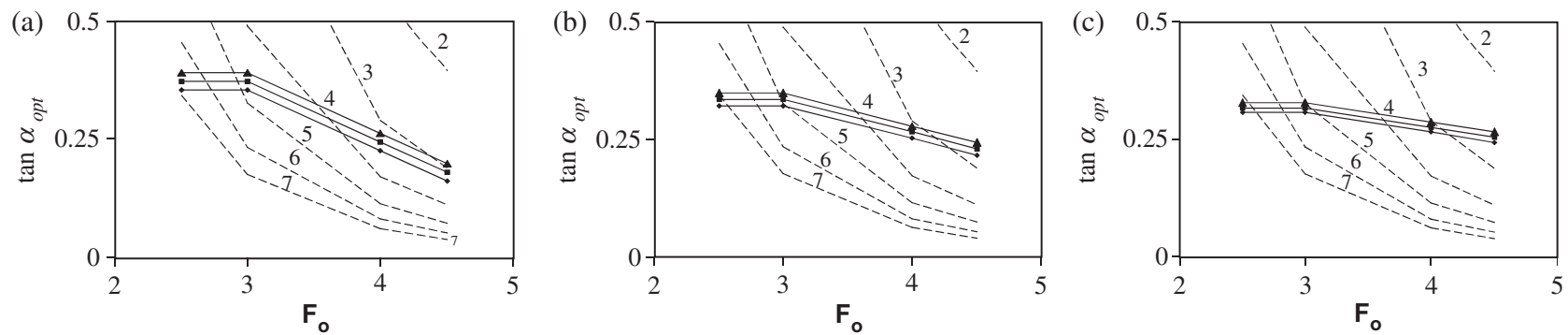

Figure 10 Variation of $\tan \alpha_{\text {opt }}$ against $\mathrm{F}_{\mathrm{o}}, d / b=$

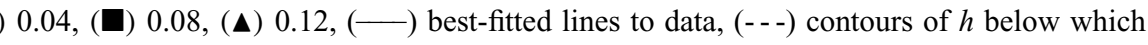
cavitation will not occur, $r_{c} / b=$ (a) 10 , (b) 15 , (c) 20
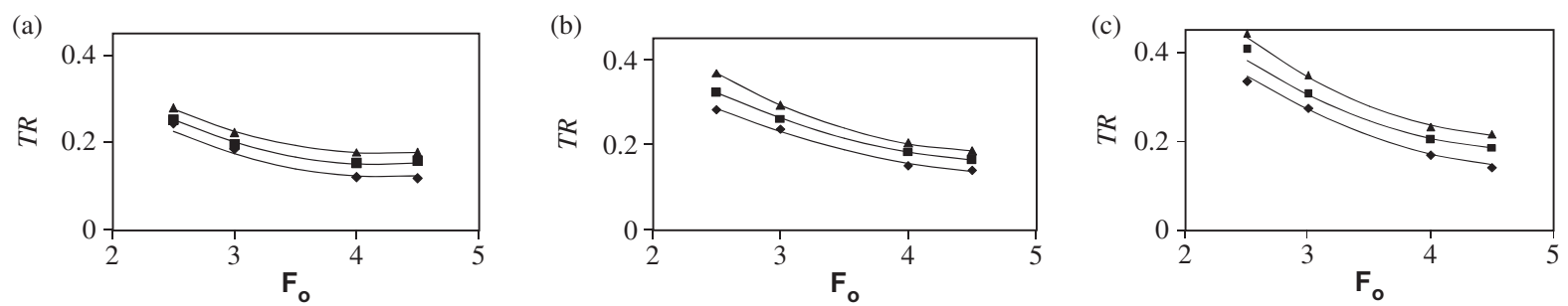

Figure 11 Variation of $T R\left(\mathrm{~F}_{\mathrm{o}}\right)$ for an optimum angle $\alpha, d / b=(\diamond) 0.04,(\mathbf{\square}) 0.08,(\boldsymbol{\Delta}) 0.12, r_{c} / b=$ (a) 10 , (b) 15 , (c) 20

\section{Convex corner and potential of cavitation damage}

In high-velocity flows, sharp-edged geometries are susceptible to cavitation damage. Around the tip of a convex corner, neglecting the gauge pressure head in close proximity to water surface, the cavitation index may be defined as

$$
\sigma=\frac{h_{a t m}-h_{v}}{V_{o}^{2} / 2 g}
$$

where $h_{\text {atm }}$ is the atmospheric pressure head and $h_{v}$ the vapour pressure head. Substituting for $h_{a t m}-h_{v} \approx 10 \mathrm{~m}$, at sea level and $25^{\circ} \mathrm{C}$, and rewriting Eq. (12) in terms of $\mathrm{F}_{\mathrm{o}}$ and $h$ gives

$$
\sigma=\frac{20}{\mathrm{~F}_{0}^{2} h}
$$

Falvey (1990) proposed an incipient cavitation index for chamfers that are away-from-the-flow as

$$
\sigma_{i}=0.83(\tan \alpha)^{0.55}
$$

At the onset of cavitation, $\sigma=\sigma_{i}$ and the depth below which cavitation will not occur is obtained from

$$
h=\frac{24}{(\tan \alpha)^{0.55 \mathrm{~F}^{2}}}
$$

In Fig. 10, depth contours from Eq. (15) are plotted by dashed lines to identify the onset of cavitation at the intersection points with solid curves. Consequently, there is a wide range of safe depths so that convex corner may be used under laboratory and field conditions without any risk of cavitation damage.

\section{Efficiency of the convex corner}

Similar to Beltrami et al. (2007) to evaluate the efficiency of a convex corner, a total reduction parameter TR is defined as

$$
\mathrm{TR}=\frac{h_{\max }-h_{\max }^{*}}{h_{\max }-h_{o}}
$$

where $h_{\max }$ is the maximum wave height without the convex corner, $h_{\max }^{*}$ is the maximum wave height in the presence of the convex corner and $h_{o}$ is the approach flow depth. Curves of $\operatorname{TR}\left(\mathrm{F}_{\mathrm{o}}\right)$ are plotted in Fig. 11 for various values of $r_{c} / b$ and $d / b$. For low Froude numbers and high $d / b$, convex corner offers a higher efficiency that increases with $r_{c} / b$. For example, using an optimum expansion angle, for $d / b=0.12, \mathrm{~F}_{\mathrm{o}}=2.5$ and $r_{c} / b=$ 20 the wave crest reduces by $45 \%$. In any case, the minimum efficiency is not below $10 \%$.

A parabolic curve may be fitted to each data set as

$$
T R=a^{\prime} \mathrm{F}_{\mathrm{o}}^{2}+b^{\prime} \mathrm{F}_{\mathrm{o}}+c^{\prime}
$$

where $a^{\prime}, b^{\prime}$ and $c^{\prime}$ are constant coefficients depending on $r_{c} / b$ and $d / b$ for different curves.

\section{Conclusions}

The performance of a small convex corner at a bend inlet is studied by the approximate Riemann solver of Roe. The negative waves originating from the corner are superimposed upon the positive waves of the outer wall. The first single-humped wave is thereby transformed to a double-humped wave of considerably reduced height. The position and height of the double-humped 
wave is a function of the radius of curvature to the bend width, the offset breadth to the bend width, the tangential angle of the expansion angle, and the approach flow Froude number. The maximum height reduction is obtained if the two humps are equal, associated with the optimum angle of the convex corner. The numerical computations for the optimum design of the convex corner in three bends reveal that the optimum angle decreases by increasing the approach flow Froude number and moderately increases by enhancing the ratio of $d / b$. Detailed studies confirm that there is a relatively wide range of safe conditions below the onset of cavitation. The efficiency of the convex corner decreases by increasing the approach flow Froude number and rises moderately by increasing the ratio of $d / b$. In the range of this study the wave height reduces by $10-45 \%$.

\section{Acknowledgements}

The financial supports of Ferdowsi University of Mashhad, Iran, during the preparation of this work, is sincerely appreciated. Special thanks are offered to Prof. Willi H. Hager, ETH Zürich, for his useful comments towards the improvement of this manuscript.

\section{Notation}

$a^{\prime}, b^{\prime}, c^{\prime}=$ constant coefficients $(-)$

$b \quad=\quad$ inlet (bend) width $(\mathrm{m})$

$d \quad=\quad$ offset width $(\mathrm{m})$

$\boldsymbol{F}(\boldsymbol{G}) \quad=\quad$ flux vector in the $x(y)$ coordinate $\left(\mathrm{m}^{2} / \mathrm{s}\right.$, $\left.\mathrm{m}^{3} / \mathrm{s}^{2}, \mathrm{~m}^{3} / \mathrm{s}^{2}\right)$

$\mathrm{F}_{\mathrm{o}} \quad=$ approach flow Froude number $(-)$

$g=$ acceleration due to gravity $\left(\mathrm{m} / \mathrm{s}^{2}\right)$

$h \quad=\quad$ flow depth, wave profile $(\mathrm{m})$

$h_{\max }=$ maximum flow depth $(\mathrm{m})$

$h_{\max }^{*}=$ maximum wave height with convex corner (m)

$h_{\text {atm }}=$ atmospheric pressure head $(\mathrm{m})$

$h_{v} \quad=\quad$ vapour pressure head $(\mathrm{m})$

$h_{o} \quad=\quad$ approach flow depth $(\mathrm{m})$

$J_{o} \quad=\quad$ Bessel function of the first kind of order zero

$l \quad=\quad$ length of convex corner $(\mathrm{m})$

$r_{c} \quad=\quad$ bend central radius $(\mathrm{m})$

$T R=$ total reduction parameter $(-)$

$u(v) \quad=\quad$ depth-averaged velocity in $x(y)$ direction $(\mathrm{m} / \mathrm{s})$

$\boldsymbol{U}=$ vector of conservative variables $\left(\mathrm{m}, \mathrm{m}^{2} / \mathrm{s}\right.$, $\mathrm{m}^{2} / \mathrm{s}$ )

$V_{o} \quad=\quad$ approach flow velocity $(\mathrm{m} / \mathrm{s})$

$\alpha \quad=\quad$ expansion angle of convex corner $(-)$

$\alpha_{\text {opt }}=$ optimum expansion angle of convex corner $(-)$

$\beta \quad=\quad$ wave $($ shock $)$ front angle $(-)$

$\beta_{1}, \beta_{2}=$ depression angles $(-)$

$\eta \quad=\quad$ maximum dimensionless depth $(-)$ $\theta \quad=\quad$ deflection angle of sidewall, central angle of bend ( - )

$\theta_{\max }, \theta_{\min }=$ location of first wave crest (trough) $(-)$

$\sigma \quad=$ cavitation index $(-)$

$\sigma_{i} \quad=\quad$ incipient cavitation index $(-)$

$\tau_{\text {ow }} \quad=$ normalized wall profile $(-)$

\section{References}

Beltrami, G.M., Repetto, R., Del Guzzo, A. (2007). A simple method to regularize supercritical flow profiles in bends. $J$. Hydraulic Res. 45(6), 773-786.

Causon, D.M., Mingham, C.G., Ingram, D.M. (1999). Advances in calculation methods for supercritical flow in spillway channels. J. Hydraulic Eng. 125(10), 1039-1050.

Chaudhry, M.H. (2008). Open-channel flow. ed. 2. Springer Science and Business Media, New York.

Chow, V.T. (1959). Open channel hydraulics. McGraw-Hill, New York.

Falvey, H.T. (1990). Cavitation in chutes and spillways. Engineering Monograph 42. USBR, Denver, CO.

Ghaeini Hessaroeyeh, M., Tahershamsi, A. (2009). Analytical model of supercritical flow in rectangular chute bends. J. Hydraulic Res. 47(7), 566-773.

Hager, W.H. (2010). Wastewater hydraulics: Theory and practice. ed. 2. Springer, Berlin.

Henderson, F.M. (1966). Open channel flow. Macmillan, New York.

Ippen, A.T. (1936). An analytical and experimental study of high velocity flow in curved sections of open channels. PhD Thesis, California Institute of Technology, Pasadena, CA.

Ippen, A.T., Dawson, J.H. (1951). Design of channel contractions. Trans. ASCE 116, 326-346.

Jan, C.D., Chang, C.J., Lai, J.S., Guo, W.D. (2009). Characteristics of hydraulic shock waves in an inclined chute contraction: Numerical simulations. J. Mech. 25(1), 75-84.

von Kármán, T. (1938). Eine praktische Anwendung der Analogie zwischen Üeberschall-Strömung in Gasen und überkritischer Strömung in offenen Gerinnen. ZAMM 18, 49-56.

Knapp, R.T. (1951). Design of channel curves for supercritical flow. Trans. ASCE 116, 296-325.

Knapp, R.T., Ippen A.T. (1938). Curvilinear flows of liquids with free surfaces at velocities above that of wave propagation. Proc. 5th Int. Congress Applied Mech., 531-536. Cambridge, Mass., John Wiley \& Sons, Inc., New York.

Leveque, R.J. (2002). Finite volume methods for hyperbolic systems. Cambridge University Press, New York.

Reinauer, R., Hager, W.H. (1996). Shockwave reduction by chute diffractor. Exp. Fluids 21(3), 209-217.

Reinauer, R., Hager, W.H. (1997). Supercritical bend flow. J. Hydraulic Eng. 123(2), 208-218.

Shamkhalchian, A.R., Jaefarzadeh, M.R. (2011). Numerical modeling of supercritical waves in bends with the 
finite volume method of Roe-TVD and appraisal of analytical assumptions. Amirkabir J. Sci. Res. 43(1), 43-50 [in Persian].

Toro, E. (2001). Shock capturing methods for free surface shallow flows. John Wiley, Chichester, New York.
Valiani, A., Caleffi, V. (2005). Brief analysis of shallow water equations suitability to numerically simulate supercritical flow in sharp bends. J. Hydraulic Eng. 131(10), 912-916.

Vischer, D.L., Hager, W.H. (1994). Reduction of shockwaves a typology. Int. J. Hydropower Dams 1(1), 25-29. 